\title{
SEXISMO Y ACADEMIA: MANIFESTACIONES DEL SEXISMO EN LA UNIVERSIDAD DE COSTA RICA
}

\author{
SEXISM AND ACADEMY: MANIFESTATIONS OF SEXISM \\ AT THE UNIVERSITY OF COSTA RICA
}

\author{
"Sexismo es falta de credibilidad por ser mujer. \\ Casi siempre hay más credibilidad a lo que vaya \\ a hacer un hombre, a lo que vaya a hacer una mujer".
}

\section{María José Chaves Groh*}

Docente consultada

RESUMEN

En el periodo 2018-2019, el Centro de Investigación en Estudios de la Mujer (CIEM) lideró una importante iniciativa para problematizar el sexismo en la Universidad de Costa Rica (UCR). Desde el enfoque cualitativo, se consultó a mujeres estudiantes, docentes y administrativas sobre esta forma de discriminación en el contexto universitario. Este artículo sistematiza las manifestaciones identificadas, donde se constata la presencia del sexismo en el ámbito universitario y su impacto en la vida laboral y académica de las mujeres.

PALABRAS CLAVE: COSTA RICA * UNIVERSIDAD * DISCRIMINACIÓN * MUJER

\section{ABSTRACT}

From the period of 2018 to 2019, the Research Center for Women's Studies (CIEM) led a major initiative to problematize sexism in the University of Costa Rica (UCR). From a qualitative approach, female students, teachers, and administrators were consulted about this form of discrimination in the university's context. This article systematizes the identified manifestations, where the presence of sexism in the university's environment and its impact on women's work and academic life is verified.

KEYWORDS: COSTA RICA * UNIVERSITY * DISCRIMINATION * WOMEN 
En América Latina, las universidades públicas continúan siendo espacios privilegiados para construir reflexiones y preguntas en torno a los avatares de nuestras sociedades. Sin embargo, tal y como señala Sagot (2018), estos centros de estudio se "enfrentan actualmente a una variedad de fuerzas sociales contradictorias que las mueven hacia una transformación profunda tanto en su estructura interna, como en sus formas de relacionarse con el resto de la sociedad" (p. 1), es decir, entre las aspiraciones de las fuerzas del mercado y la búsqueda del bienestar social, existe una fuerte disputa para definir quiénes son las voces autorizadas y cuáles son las preguntas pertinentes para generar conocimiento desde los centros de educación superior.

Esta disputa no solo se evidencia en la relación de las universidades con las sociedades, sino también a lo interno de las universidades mismas. En este sentido, una de las fuerzas contradictorias que Sagot (2018) destaca es el régimen sexista que hace parte de las estructuras universitarias y que se sostiene con la complicidad de diferentes actores para perpetuar - a través de diferentes mecanismos- la exclusión de las mujeres.

En este sentido, la definición de sexismo que propone Morgade (2001, como se citó en Araya, 2003) resulta sumamente pertinente, entendiéndolo como "una práctica que tiende a encorsetar a las personas en parámetros impuestos" (p. 43). De esta forma, el sexismo utiliza el sexo de las personas como un factor de organización social que "establece un lugar de subordinación para las mujeres y reclama una supremacía para los varones" (Mingo y Moreno, 2017, p. 572). De acuerdo con Araya (2003):

El sexismo utiliza al sexo como criterio de atribución de capacidades, valoraciones y significados creados en la vida social, ordenando a la realidad con los cajones "esto es lo femenino" "esto es lo masculino".

La diferenciación sexista afecta a las mujeres $y$ a los hombres. Sin embargo, en el sexismo la diferencia se coloca en una escala jerárquica que termina por sostener el argumento que el diferente, en este caso las mujeres, son inferiores (p. 43).

De acuerdo con Flores y Espejel (2015, como se citó en Mesa, 2019), "el sexismo implica ejercer el poder para mantener en situación de inferioridad, subordinación, explotación al sexo opuesto, al valorarla negativamente en función de su sexo y en general se dirige hacia las mujeres" (pp. 6-7).

Sin embargo, diferentes autoras coinciden en plantear que el sexismo no se manifiesta de la misma forma en todos los espacios, es decir, "cambia de ropajes, se metamorfosea $y$, en ciertos ambientes - sobre todo en aquéllos permeados por la mentalidad de lo "políticamente correcto"-, se vuelve difícil de identificar" (Mingo y Moreno, 2017, pp. 582-583).

Concretamente, en la Universidad de Costa Rica (UCR), las mujeres han conseguido atravesar las fronteras que las alejaban de las aulas universitarias y han irrumpido con fuerza en áreas del conocimiento dominadas históricamente por los hombres, no obstante, en este espacio "políticamente correcto", de acuerdo con Mingo y Moreno, el sexismo ha mutado sus formas de exclusión para reafirmar que el camino del crecimiento académico y laboral no fue diseñado para las mujeres, por lo tanto, quienes insistan en transitar por este, lo deben hacer siguiendo las reglas predefinidas. En este sentido, Araya (2003) plantea que:

(...) el hecho de que las mujeres accedan cada vez más a la igualdad formal (...) no supone que tengan las mismas oportunidades que los hombres, sino más bien que las formas de discriminación se tornan más sutiles y menos evidentes, persistiendo diversas manifestaciones del sexismo que es necesario desentrañar (p. 43).

En este contexto, en el año 2018, el Centro de Investigación en Estudios de la Mujer (СIEM) ${ }^{1}$ impulsó el proyecto Visibilizar y transformar

1 Este centro de investigación es parte de la Universidad de Costa Rica. 
prácticas sexistas y discriminatorias en la Educación Superior, con el propósito de visibilizar el régimen sexista que prevalece en la UCR, problematizar sus estrategias de reproducción y pensar colectivamente estrategias de transformación.

Entre otras, una de las principales tareas que desarrolló este Proyecto fue el diseño de una campaña de sensibilización que diera cuenta de diferentes expresiones de sexismo que enfrentan mujeres estudiantes, docentes y administrativas; así como, las implicaciones que esta forma de exclusión tiene en sus proyectos laborales y académicos. El diseño de esta campaña se fundamentó en los testimonios que brindaron estas mujeres a través de una serie de consultas realizadas en diferentes sedes de la UCR.

En este artículo, se analizan los testimonios recogidos, las demandas y propuestas que las mujeres consultadas formularon para visibilizar y erradicar esta forma de discriminación en el ámbito universitario.

\section{RUTA METODOLÓGICA PARA REALIZAR LAS CONSULTAS}

La ruta metodológica planteada se definió desde el enfoque cualitativo, esto con el fin de caracterizar y comprender las manifestaciones del sexismo en la UCR. El primer paso en este proceso fue realizar una revisión bibliográfica a nivel nacional e internacional, que permitiera conceptualizar el sexismo e identificar experiencias de otros centros de educación superior donde se estudiaron cómo se manifiesta esta forma de discriminación en sus propios espacios.

Concluida la revisión bibliográfica se procedió a diseñar tres guías de grupo focal, una dirigida a mujeres estudiantes, otra a docentes $y$ otra a administrativas, sin embargo, las tres guías se enfocaron en el mismo objetivo y compartían los mismos temas.

Para esta recolección de información se privilegió la técnica de grupo focal. Esta técnica permite que un pequeño grupo de personas (se recomienda entre 5 y 7) con características similares, por ejemplo, siendo todas las participantes estudiantes o todas docentes, puedan conversar en profundidad sobre un tema específico (Buss Thofehrn et al., 2013), en este caso, las diversas maneras en qué se manifiesta el sexismo en el espacio universitario.

La aplicación de los grupos focales se llevó a cabo en el segundo semestre del año 2018 y contó con la participación de 42 mujeres distribuidas en siete consultas: una con estudiantes y otra con administrativas en la Sede del Atlántico, una con estudiantes en la Sede del Caribe, una con docentes en la Sede de Guanacaste, una con estudiantes y otra con docentes en Occidente, $y$ otra con estudiantes en la Sede Central Rodrigo Facio.

La convocatoria de las participantes se realizó por medio de funcionarias con las cuales el CIEM ya tenía coordinaciones previas en las diferentes sedes, así como de representantes de asociaciones de estudiantes $y$ de la Federación de Estudiantes de la Universidad de Costa Rica (FEUCR). Con los insumos generados en las consultas, se procedió a desarrollar este artículo de análisis y a identificar los mensajes fuerza que posteriormente se posicionaron en productos de comunicación: afiches, imágenes para redes sociales, pines, calcomanías, cuñas de radio y videos.

\section{LOS HALLAZGOS}

A continuación se presentan diferentes manifestaciones de sexismo narradas por las participantes en los grupos focales, manifestaciones que van desde expresiones evidentes, ampliamente documentadas como las brechas de género o el hostigamiento sexual, hasta otras más sutiles, mimetizadas para permanecer y reproducirse en un entorno académico "políticamente correcto", por ejemplo, la generación de parcelas de conocimiento, donde parece que por una senda transita la ciencia y por otra los estudios de género.

Cabe destacarse que todas las participantes en todos los grupos focales compartieron al menos una situación de sexismo que han vivido en la Universidad, lo cual revela dos elementos fundamentales para problematizar el sexismo en este espacio académico, primero, esta forma de discriminación es reconocida por las mujeres 
universitarias y segundo, la Universidad no es un espacio ajeno a esta forma de discriminación.

Además de ejemplificar de diferentes maneras el sexismo en la UCR, las participantes hablaron sobre las afectaciones de esta forma de discriminación en sus condiciones laborales $y$ académicas, $y$ en sus expectativas de crecimiento; así como, las propuestas y las recomendaciones que le formulan a la Universidad para visibilizar el sexismo y generar transformaciones que apunten a generar un entorno más democrático y de mayor igualdad entre hombres $y$ mujeres.

\section{¿CÓMO SE MANIFIESTA EL SEXISMO?}

"Me pregunto para qué proponer, para qué tener ideas, para qué pensar si mi voz de mujer no la escuchan" (estudiante consultada, comunicación personal, 4 de septiembre de 2018).

1) Segregación horizontal. Probablemente esta sea la manifestación más documentada del sexismo en el ámbito educativo. Maubrigades (2020) define la segregación horizontal como "la distribución desigual de mujeres y hombres en los diferentes sectores de la economía o en las diferentes ocupaciones" (p. 10). Esta segregación genera una alta concentración de hombres $y$ de mujeres estudiando o trabajando en áreas tradicionalmente asignadas a su sexo, por ejemplo, las mujeres en las ciencias sociales y los hombres en las ciencias exactas.

La gente categoriza por sexo las carreras desde Ciencias del Movimiento Humano, las ingenierías, informática. Y a una lo ven raro cuando está en esos espacios (estudiante consultada, comunicación personal, 2 de noviembre de 2018).

2) Minimizar sus propuestas. En las consultas realizadas con docentes, administrativas $y$ estudiantes se identifica con facilidad expresiones de sexismo que son comunes entre estos diferentes colectivas, desde minimizarlas por su condición de mujeres, hasta menospreciar sus opiniones o propuestas.
Cuando yo quiero un tema lo defiendo, insisto, e insisto hasta que ya me den pelota, a veces tengo que decirlo 5, 6 ó 7 veces, pero lo que diga un compañero a la primera todo el mundo le da pelota, a mí no (estudiante consultada, comunicación personal, 4 de septiembre de 2018).

[Nombre de la carrera] es una carrera asociada al cuido, lo cual está relacionado con el hecho de que sea una carrera profundamente feminizada. Sin embargo, cuando un hombre [de la misma carrera] habla, la audiencia calla porque el hombre está hablando (docente consultada, comunicación personal, 6 de septiembre de 2018).

Lo manifiestan a veces en forma no verbal, pero muy poco sutil (...) en una reunión, donde digamos una de nosotras es la que lleva la propuesta, a veces por lo general con su lenguaje corporal demuestran eso, pereza y apatía de una forma muy poco sutil con sus gestos nos anulan (administrativa consultada, comunicación personal, 2 de noviembre de 2018).

3) Los temas de mujeres. Las experiencias narradas por las estudiantes reflejan que la segregación y la descalificación de los temas relacionados con las desigualdades de género o con el feminismo, se empiezan a gestar desde las aulas universitarias, a partir de las opiniones de compañeros de clase o, incluso, de sus propios docentes.

Cuando queremos trabajar un tema sobre feminismo en clase o como trabajo de investigación, los compañeros dicen "ay pero de eso ya se ha hablado mucho, hablemos de algo más" o "eso es muy delicado, mejor no hagamos polémica" (estudiante consultada, comunicación personal, 4 de septiembre de 2018). 
Exponiendo la tesina sobre hostigamiento sexual callejero, los profes hombres interrumpían para decir que a los hombres también los acosan y contaban sus anécdotas durante nuestra exposición (estudiante consultada, comunicación personal, 2 de noviembre de 2018).

En un artículo reciente, Guizardi, Gonzálvez y Stefoni ${ }^{2}$ llaman la atención sobre el impacto que tiene en las personas investigadoras esta compartimentación del conocimiento científico, ya que los análisis de género quedan confinados a las revistas especializadas en mujeres, feminismo o estudios de género, aplicando una censura previa que impide posicionar estos temas en revistas académicas de amplios campos temáticos como las ciencias sociales, la demografía o la historia.

(...) los debates críticos de género están siendo progresivamente arrinconados como temas que no se pueden publicitar $y$ publicar sino en revistas feministas. Esta censura temática, que extrapola los supuestos lineamientos meritocráticos que definen el modo de evaluación de las revistas, tiene consecuencias concretas para las y los investigadores que trabajamos con estos temas: al dificultar, ralentizar $y$ a veces frustrar nuestras publicaciones, nos empujan a la periferia del sistema de productividad científica (...) (Guizardi, Gonzálvez y Stefoni, 2019, en evaluación).

4) Los hombres insisten en interpretar lo que nosotras queremos decir. Mansplaining es el término anglófono que se ha empleado en los últimos años para nombrar una práctica bastante conocida por las mujeres de todos los tiempos, la explicación que los hombres pretenden darles a ellas mismas sobre lo que ellas piensan, dicen o sienten, incluso hasta del conocimiento que ellas mismas generan.

2 El documento se encuentra en proceso de evaluación para publicación.
Sobre esta forma de subalternización, las estudiantes consultadas también tuvieron mucho que decir, sobre todo, desde sus propios compañeros de clase o con quienes militan en el movimiento estudiantil.

En [nombre de la carrera] pasa mucho que una dice algo y algún compañero interviene $y$ dice no, no lo que usted quiso decir es esto y una es como no, no, yo quise decir lo que yo dije (estudiante consultada, comunicación personal, 4 de septiembre de 2018).

En todos los espacios políticos en los que he participado, siempre me he topado compañeros que son falsos feministas hablando de las mujeres como si tuvieran propiedad de lo que sentimos (estudiante consultada, comunicación personal, 4 de septiembre de 2018).

5) Estereotipos de género. Los estereotipos son "las etiquetas o signos que ponemos a las personas según pertenezcan a un sexo o a otro. Los definimos como los estereotipos culturales de hombres y mujeres que señalan como tenemos que actuar en nuestra vida cotidiana" (Vilá y Tordera, 2011 como se citó en López, 2020, p. 137).

En las consultas realizadas, las estudiantes enumeraron una amplia lista de situaciones en las cuales se han enfrentado a los estereotipos sexistas que históricamente las han excluido de espacios académicos y profesionales. En este sentido, algunas dicen haberse sentido infantilizadas, cuestionadas en sus capacidades intelectuales $y$ deportivas o en sus destrezas para el uso de las tecnologías, sobre todo por parte de sus profesores, pero también por parte de sus compañeros.

A mí me ha pasado con la tecnología (...) $y$ muchas veces pasa que si un profesor o profesora no sabe poner el volumen de los parlantes entonces dice "¿hay algún muchacho que sepa hacer esto?” (estudiante consultada, comunicación personal, 4 de septiembre de 2018). 
No importa cuántas veces un hombre haga una canasta [en un juego de baloncesto] o un buen movimiento que siempre le van a felicitar, pero lo hago yo y me dice "Uy mae vio que guaba"3 (estudiante consultada, comunicación personal, 2 de noviembre de 2018).

Uno de los profesores de [nombre de la carrera] trata a las mujeres de "niñas" $y$ a los hombres por su nombre, es el mismo profesor que les toma fotos a las estudiantes y las agrega al Facebook y les comenta sus fotos (estudiante consultada, comunicación personal, 2 de noviembre de 2018).

6) Hostigamiento sexual. De acuerdo con la normativa nacional y el Reglamento contra el Hostigamiento Sexual de la Universidad de Costa Rica (31 de agosto de 2020), se entiende por acoso u hostigamiento sexual toda conducta sexual indeseada por quien la recibe, reiterada $y$ que provoque efectos perjudiciales en los siguientes casos:

a) Condiciones materiales de empleo o de docencia.

b) Desempeño y cumplimiento laboral o educativo.

c) Estado general de bienestar personal.

También se considera acoso sexual la conducta grave que, habiendo ocurrido una sola vez, perjudique a la víctima en cualquiera de los aspectos indicados (artículo 3, Reglamento contra el Hostigamiento Sexual, UCR).

El hostigamiento sexual es probablemente una de las formas de violencia más discutida $y$ visibilizada en la UCR. En este sentido, desde 1997, la UCR cuenta con un reglamento amparado en la normativa nacional e internacional que delimita este comportamiento, norma el proceso de denuncia y establece sanciones para

3 Sinónimo de casualidad, golpe de suerte (costarriqueñismo). quien incurre en estas prácticas. Además, en el CIEM existen dos equipos de trabajo interdisciplinario, uno está a cargo de los procesos de sensibilización y capacitación dirigidos a la comunidad universitaria $y$, el otro equipo, al acompañamiento legal y emocional de las personas que interponen denuncias por hostigamiento sexual.

A pesar de estos indiscutibles avances, los testimonios de las estudiantes consultadas dan cuenta de que aún no se ha conseguido erradicar esta forma de violencia sexista.

El profesor de [nombre de la materia que imparte] le dijo a una amiga que le mandara fotos de sus mejores partes (estudiante consultada, comunicación personal, 2 de noviembre de 2018).

Le cerró la puerta de clase y le dijo que porque no se veían un día, porque le veía medio triste y él la podía animar (estudiante consultada, comunicación personal, 2 de noviembre de 2018).

Hay muchachas que tienen que poner pupitres entre ellas y los profes, para evitar que los profesores se les acerquen o las toquen. Han tenido que desarrollar estrategias para ponerse a salvo (estudiante consultada, comunicación personal, 2 de noviembre de 2018).

7) La minorización de la violencia contra las mujeres. Descalificar a las mujeres, ridiculizar su sentir, minimizar la violencia en su contra o igualarla a la violencia que enfrentan los hombres, son estrategias sexistas utilizadas tanto por docentes como por estudiantes hombres, para discriminar $y$ violentar a las mujeres en la Universidad.

En una clase de derecho penal he escuchado compañeros que dicen "a mí no me parece que haya una ley que proteja más a las mujeres en casos de asesinatos por crímenes pasionales porque igual le puede pasar a los hombres" y 
una sí, puede pasarle a los hombres pero la tasa es demasiado baja y casi nunca pasa por crímenes pasionales y cuando los matan, usualmente son hombres matando otros hombres (estudiante consultada, comunicación personal, 4 de septiembre de 2018).

Otra estrategia utilizada con frecuencia que deja a las mujeres en una situación de profunda indefensión y duda frente a sí mismas es cuando los ofensores ocultan tras un velo de broma sus comentarios sexistas, es decir, no se responsabilizan por los actos o los comentarios sexistas que han realizado porque son conscientes de que en un espacio "políticamente correcto" esa conducta podría ser reprochable por algunas personas en su entorno, por lo tanto, lo hacen igual pero lo minimizan descalificando o ridiculizando a las mujeres por molestarse, quejarse o denunciar la situación.

Una vez un colega me hizo un chiste que me dolió muchísimo, porque además de ser mujer soy pequeña, estábamos en una reunión importante de Consejo, había un baño que estaba muy alto, y el compañero me dijo "Compañera ¿cómo hizo, le pusieron un banquito para ir al baño?", yo me devolví y le respondí "Disculpe compañero, no, a mí nunca me han tenido que poner un banquito ni para ir al baño, ni para tener un doctorado, ni para estar aquí" (docente consultada, comunicación personal, 6 de septiembre de 2018).

Cuando le hacemos frente a algún comentario de compañeros, nos dicen "ay, como usted es una mujer herida por el patriarcado" y el resto se ríe (estudiante consultada, comunicación personal, 4 de septiembre de 2018).

En esta dinámica entra en juego otra estrategia sexista que ha sido más estandarizada a nivel social, convencer a las mujeres de que ellas no pueden estar juntas, trabajar juntas o ser solidarias entre sí. Este mandato de desconfianza entre mujeres debilita sus oportunidades de encuentro y diálogo, sus oportunidades para poner en común las formas de discriminación que enfrentan, sus mecanismos de defensa $y$, sobre todo, validarse entre ellas para fortalecer su autoconfianza y seguridad.

A veces pensaba que estoy loca $y$ tengo que dejar de ser exagerada, tengo que dejar de pensar en eso, pero luego, cuando veo otras chicas en otros espacios, yo digo es que no, yo no estoy loca ni me lo estoy inventando, es que esto nos pasa por el cuerpo todos los días (estudiante consultada, comunicación personal, 4 de septiembre de 2018).

Las diferentes experiencias narradas por las participantes de los grupos focales, reflejan cómo se fortalece la estructura social que subordina a las mujeres $y$ a lo femenino frente a los hombres y lo masculino, recordándole a las mujeres - y a los hombres- cuál es el lugar que socialmente les corresponde, en palabras de Mingo y Moreno (2017), "el acto de habla sexista (re)instaura el orden de género" (p. 580).

\section{CONSECUENCIAS EN LA VIDA ESTUDIANTIL, ACADÉMICA, LABORAL Y PERSONAL}

A pesar de la intensa lucha que las mujeres están dando en la Universidad de Costa Rica para denunciar $y$ defenderse de estas formas de discriminación y violencia, lo cierto es que el propósito de excluirlas se cumple a cabalidad.

En el ámbito estudiantil, se refleja en diferentes dimensiones, por ejemplo, en la participación política. En los últimos 50 años, el mayor órgano de representación estudiantil, la Federación de Estudiantes de la Universidad de Costa Rica (FEUCR) ha tenido 51 representantes (se han dado algunas reelecciones), sin embargo, de ese total 42 han sido hombres y solo 9 han sido mujeres.

Los relatos compartidos por las estudiantes participantes de las consultas, evidencian exclusiones e inseguridades que se mueven en la política estudiantil con relación a la participación de las mujeres: 
En una reunión de Representación Estudiantil un estudiante dijo que la Mesa tenía tantos problemas porque quienes la estábamos dirigiendo éramos mujeres (estudiante consultada, comunicación personal, 31 de agosto de 2018).

Siempre me limité mucho a intentar hacerlo [tener aspiraciones políticas] porque, aunque fuera una estupidez lo que ellos estuvieran proponiendo, siempre es más importante que lo que yo esté aportando (estudiante consultada, comunicación personal, 4 de septiembre de 2018).

Otro ámbito de graves consecuencias para las estudiantes es la distribución de horas para asistir a docentes. Las horas estudiante $\mathrm{u}$ horas asistente representan una valiosa oportunidad, ya que en términos económicos les garantiza el acceso a una beca y en términos profesionales les permite adquirir experiencia $y$ reconocimiento frente a docentes que posteriormente podrían recomendarles para un trabajo dentro o fuera de la Universidad.

Si bien, la normativa institucional exige que se hagan concursos abiertos para la asignación de estas horas, al momento de realizar la selección cada docente establece sus propios criterios.

Un profesor me dijo que las horas becas con él solo eran para hombres por que había que andar jalando el equipo y cosas que solo hacen los hombres (estudiante consultada, comunicación personal, 27 de agosto de 2018).

Hay un profe que sólo contrata como asistente a las que considera bonitas, para el gusto de él (estudiante consultada, comunicación personal, 27 de agosto de 2018).

Un profe nunca le daba asistencias a mujeres ya que decía que eran labores de hombres, pero le rogué como tres veces $y$ al final me dio las horas. Ahora es muy accesible y contrata a hombres $y$ mujeres. Dice que yo lo hice ver las cosas diferente (estudiante consultada, comunicación personal, 27 de agosto de 2018).

Para las docentes, la situación no es más alentadora. El modelo de incentivos profesionales de la Universidad se construye sobre la base de lo que Rodríguez (2007) denomina un "modelo de trabajador ideal" (p. 231), que dista mucho de la realidad de la mayoría de las mujeres. Al respecto, la autora agrega otro concepto fundamental, domesticidad entendida como el proceso mediante el cual se especializa socialmente a las mujeres para que asuman las tareas de cuidados (Rodríguez, 2007).

Siguiendo a Rodríguez (2007), la domesticidad se sostiene sobre de la base de dos pilares, un "trabajador ideal que se ocupa a tiempo completo, e incluso trabaja horas extra, y que destina muy poco tiempo a las tareas de mantenimiento físico del hogar y cuidado de las personas dependientes" (p. 231), y la marginalización de quienes desarrollan las tareas de cuidados.

De esta manera, así como las fábricas esperan personas trabajadoras dispuestas a vender su fuerza de trabajo de forma ilimitada, la Universidad espera personal académico dispuesto a generar publicaciones $y$ a avanzar en grados académicos de forma ilimitada. En este sentido, respondiendo al segundo pilar de la domesticidad, ni las fábricas ni las Universidades asumen que la reproducción de la fuerza de trabajo de unos y otros es posible gracias al trabajo invisible que socialmente se asigna a las mujeres. Por lo tanto, cuando las mujeres trabajan en las fábricas o en las universidades, deben responder a cabalidad con el modelo de "trabajador ideal" descrito anteriormente, pero sin descuidar sus tareas de cuidados (su hogar, su familia, su matrimonio, sus hijas e hijos) porque de ser así será fuertemente sancionada y culpabilizada socialmente.

Recientemente, Mesa (2019) realizó un análisis cualitativo que da cuenta de las dificultades que enfrentan las mujeres para crecer en el mundo académico. Entre sus 
conclusiones destaca que "la conciliación entre el trabajo como docentes y la vida doméstica es complicada $y$ depende muchas veces de lograr el apoyo de otras mujeres, ya sean familiares o empleadas domésticas" (p. 5). Además, a pesar de pertenecer a estratos sociales con alto nivel educativo, en las familias de las docentes, sus carreras no se perciben como algo importante por lo que han debido "renunciar a becas de doctorado, esperar a que sus familias se duerman para levantarse a escribir porque sus parejas, también profesionales, entienden que la crianza de los hijos e hijas y el trabajo doméstico les corresponden a las mujeres" (Mesa, 2019, p. 28).

Otro aspecto que destaca Mesa en su artículo son las tareas que se asignan a las mujeres en la misma Universidad, tareas que justamente reproduciendo el modelo sexista son menos valoradas, menos reconocidas en términos académicos y menos remuneradas, por ejemplo, la representación en comisiones, el trabajo administrativo y la acción social. Esto se traduce en una carga de trabajo importante para las docentes que limita aún más sus oportunidades de crecimiento en la escala universitaria.

El Reglamento de Régimen Académico (...) no considera las diferencias que el género introduce en las posibilidades de realizar el trabajo universitario, pues hace énfasis en la investigación, en los doctorados $y$ en las publicaciones, $y$ da escasa relevancia a la acción social y a la docencia (Mesa, 2019, p. 28).

Al respecto, Sagot (2018) señala que estudios recientes evidencian que:

(...) los profesores hombres, dedicaban un $37 \%$ de su tiempo a actividades de investigación, (la actividad que más les brindará oportunidades de avanzar en sus carreras $y$ de obtener reconocimientos $y$ prestigio), y solo un $20 \%$ a actividades de servicio como comisiones $y$ trabajo administrativo. Las mujeres, por el contrario, solo dedican un $20 \%$ de su tiempo a la investigación y más del 30\% a las actividades de servicio, las que menos posibilidades les brindan de avance (p. 10).
Lo analizado por Mesa y Sagot hace eco en la palabra de las docentes consultadas. En sus relatos, ellas confirman el impacto que tiene la imposibilidad de conciliar las tareas de cuidados que realizan en sus hogares con su desempeño profesional, debido a que sienten una sobre exigencia por parte de la Universidad, como si tuvieran que pagar una factura por ser mujeres, docentes, madres o responsables de cuidados, o incluso por intentar un proyecto de vida que trascienda el modelo de "trabajador ideal" descrito anteriormente.

(...) el trabajo voluntario o el menos valorizado es usualmente recargado en las mujeres docentes, mientras las funciones o tareas más reconocidas son realizadas por los hombres docentes, a ellos si se les reconocen salarialmente sus contribuciones y a nosotras no. Es decir, existe una sobre carga de trabajo no reconocido en las mujeres docentes, al igual que ocurre en las casas (docente consultada, comunicación personal, 6 de septiembre de 2018).

Cuando queremos sacar un posgrado, la estructura institucional nos golpea porque nos piden las mismas condiciones que a los hombres $y$ no estamos en las mismas condiciones de tiempo, ni de libertad que los hombres (...) son contados los hombres que asumen los cuidos, la crianza y labores domésticas (docente consultada, comunicación personal, 6 de septiembre de 2018).

El sexismo me limita hasta el decidir tener un plan de vida con mi pareja. (docente consultada, comunicación personal, 6 de septiembre de 2018).

A estas formas de sexismo se suman las dificultades que enfrentan las mujeres docentes para optar por becas o especializaciones, ya que - especialmente quienes están en edad reproductiva - son vistas como potenciales madres y se asume que la maternidad les resta capacidad 
productiva. En este sentido, la Universidad y el mundo académico lejos de generar condiciones para conciliar la maternidad y la vida académica, procura desestimular la participación de las mujeres, apelar al agradecimiento que ellas deben sentir por permitírseles trabajar en un centro de educación superior y recalcar que ese agradecimiento se debe retribuir asumiéndose "trabajadoras ideales", o bien, minimizando sus logros.

Las dos primeras preguntas que me hicieron cuando concursaba para una beca en el exterior fueron que si estaba casada y que si tenía hijos. Me sorprendió habérmela ganado, sin embargo, lo primero que me dijeron fue "recordá que tenés que cuidarte, ojalá que no te enamores y no tengas hijos allá" (docente consultada, comunicación personal, 7 de septiembre de 2018).

Luego de mucho tiempo de estudiar fuera, el día que regreso y me incorporo en el trabajo había una Asamblea Ampliada. Yo estaba muy emocionada de ver a todos de nuevo. Lo primero que dicen después de mi nombre fue "como pueden ver viene más delgada." No dijeron nada de mi doctorado, ni de mi recorrido (docente consultada, comunicación personal, 7 de septiembre de 2018).

Para conseguir becas o trabajos me ha tocado decir "no se preocupen, no tengo hijos, estoy divorciada y soy igual de fértil que una piedra" (docente consultada, comunicación personal, 7 de septiembre de 2018).

(...) una compañera que iba a cambiar de puesto pero estaba embarazada, y llegó a sentir temor que no le dieran el puesto debido al embarazo. Yo habría pensado lo mismo, a estas alturas estamos igual, existen muchas cosas que no cambian (administrativa consultada, comunicación personal, 2 de noviembre de 2018).
Las narraciones compartidas por las participantes se corresponden con los datos oficiales que dan cuenta de la participación de hombres y mujeres en las diferentes categorías académicas. De acuerdo con un Informe de Brechas de Género en Recursos Humanos de la UCR, en la categoría de Instructor (categoría de ingreso para las y los docentes en Régimen Académico), los hombres representan el 63,6\% $y$ las mujeres $36,4 \%$. En la categoría de Catedrático (nivel más alto para las y los docentes), estos datos se mantienen casi intactos, $63,4 \%$ son hombres mientras que el $36,6 \%$ son mujeres (Universidad de Costa Rica, 2017).

Estas realidades son un triste reflejo de lo que plantea Sagot:

(...) conforme avanzamos en el siglo XXI es increíble que una de las instituciones más progresista e ilustrada haya cambiado tan poco en su estructura y organización, $y$ que siga reproduciendo una forma de hacer las cosas que responde a las características y necesidades del varón tradicional, entendido como el sujeto que tiene control y predominio del espacio público porque tiene todas sus necesidades satisfechas en el espacio privado. Es decir, el modelo de la universidad se ha construido sobre la base del hombre proveedor y para tener éxito, las mujeres o tiene que convertirse en hombres simbólicos o tratar de asemejarse lo más que puedan a ese modelo masculino, o tenemos que soportar abusos (2018, p. 8).

En términos personales, el sexismo también pasa factura. De acuerdo con los relatos compartidos por las participantes de las consultas, los comentarios y las acciones sexistas que enfrentan cotidianamente en el quehacer universitario han conseguido el propósito de afectar su imagen, su confianza y su percepción sobre sí mismas.

A mí me ha costado descubrir mi voz y decir no, yo soy igual que ustedes, tengo la misma capacidad y también puedo aportar (estudiante consultada, comunicación personal, 4 de septiembre de 2018). 
Cuando estoy en clases $y$ salen este tipo de comentarios a veces no me doy cuenta en el momento, $y$ me da cólera conmigo misma por no responder en el momento, entonces siento que tengo que estar alerta para saber qué responder (estudiante consultada, comunicación personal, 4 de septiembre de 2018).

Dicen que qué difícil trabajar con mujeres porque somos complicadas, como que todo nos molesta. Llega un punto donde una se cuestiona si será cierto que tengo algo malo (administrativa consultada, comunicación personal, 2 de noviembre de 2018).

Para las docentes, la afectación se traduce en incertidumbre laboral, dificultades para crecer en términos académicos, desmotivación y serias afectaciones en su salud física y emocional:

(...) represalias laborales, falta de nombramientos [funcionarias en condición de interinas], horarios difíciles (...) tardan días en firmar oficios (docente consultada, comunicación personal, 7 de septiembre de 2018).

Las docentes que se pensionan, lo hacen con un estado muy deteriorado de salud. Es la cosa de legitimar el trabajo fuera de la casa, tener que convencer con mayor carga laboral que sos valiosa (docente consultada, comunicación personal, 6 de septiembre de 2018).

Es que es un impacto emocional, es un desgaste, sabiendo que al presentar algo [una queja o una denuncia] seguro van a reaccionar mal, de forma sexista. Entonces una le invierte toda esa energía a estar atenta a lo que una tiene que hacer, además de la autorregulación para no reaccionar mal ante los gestos de apatía y de desinterés de los compañeros (administrativa consultada, comunicación personal, 2 de noviembre de 2018).

\section{¿QUÉ ESPERAN DE LA UNIVERSIDAD?}

Lo dicho anteriormente evidencia que el sexismo en la UCR está presente. Pese a los enormes esfuerzos que las mujeres realizan para continuar con sus carreras, sea como estudiantes o como funcionarias, lo cierto es que aún están lejos de conseguir el reconocimiento de su palabra, el debate franco de sus ideas y condiciones justas para destacar en sus áreas.

Es muy duro para nosotras enfrentarnos todos los días en las clases, que se supone es un lugar seguro $y$ donde la $\mathrm{U}$ se supone es una burbuja para que nosotras crezcamos como personas, y que la Universidad no nos esté ayudando ni apoyando (estudiante consultada, comunicación personal, 4 de septiembre de 2018).

En este contexto, ellas le plantean a la Universidad una serie de expectativas y demandas en diferentes ámbitos, desde el posicionamiento explícito de no seguir siendo cómplice de prácticas sexistas, hasta generar normativa y procesos de sensibilización coherentes con este planteamiento.

Con relación a lo normativo, según se indicó anteriormente, la UCR cuenta con un reglamento sobre hostigamiento sexual. Si bien, aún es necesario impulsar mejoras en el propio reglamento y su implementación, lo cierto es que su existencia representa un mecanismo importante para tipificar el hostigamiento $y$ sancionarlo. Al momento de realizar este estudio, la Universidad aún no contaba con una norma para sancionar esta forma de discriminación ${ }^{4}$, por lo tanto, las docentes, estudiantes y funcionarias administrativas consultadas urgían un reglamento que permita tipificar, prevenir y sancionar el sexismo en el ámbito universitario.

Ampliación o reglamento complementario contra el hostigamiento laboral y hostigamiento laboral sexista que nos permitiera denunciar (administrativa

El 16 de abril del año 2020, el Consejo Universitario aprobó el Reglamento de la Universidad de Costa Rica en contra de la discriminación. 
consultada, comunicación personal, 2 de noviembre de 2018).

Campaña para visibilizar que el sexismo existe en profesores, administrativos y estudiantes e interiorizarla en todas las carreras (estudiante consultada, comunicación personal, 4 de septiembre de 2018).

Campañas de información para que se sepan cuáles y cómo son los procesos para denunciar y acompañar casos de sexismo y hostigamiento sexual (estudiante consultada, comunicación personal, 2 de noviembre de 2018).

De forma paralela, las participantes señalan la importancia de sensibilizar a toda la comunidad universitaria sobre el sexismo, esto como una estrategia de prevención, pero también de acompañamiento a las mujeres que requieren hablar sobre el tema, buscan información o deciden interponer una denuncia.

(...) hablar de estos temas, abrir estos espacios a lo interno, porque nosotros a nivel administrativo a veces tenemos muy poca capacitación en cuestiones personales y relaciones humanas (administrativa consultada, comunicación personal, 2 de noviembre de 2018).

A veces una no dice nada por miedo a ser juzgada. Realmente no son cosas pequeñas. ¿Cómo va a acabar esto si seguimos con ese miedo y sin respeto? (estudiante consultada, comunicación personal, 27 agosto de 2018).

En la misma línea, las estudiantes y las funcionarias destacan la importancia de generar espacios de sensibilización que alcancen a los órganos de la FEucr, el Sindicato de Trabajadores de la Universidad de Costa Rica (SINDEU) $y$ otras entidades gremiales que existen en esta casa de estudios.

\section{REFLEXIONES FINALES}

A partir de los testimonios recogidos en los grupos focales, se plantean las siguientes reflexiones finales:

El sexismo es una forma de discriminación reconocida por las participantes. Todas las participantes de todos los grupos focales narraron situaciones que identifican como manifestaciones de machismo o de sexismo en la Universidad.

El sexismo es un mecanismo vigente para estructurar la subordinación de las mujeres y lo femenino en espacios académicos. A pesar de que la Universidad es un espacio privilegiado para la reflexión, la crítica y la problematización de las desigualdades, aún hoy el sexismo establece las reglas a partir de las cuales hombres $y$ mujeres se relacionan con este espacio, como centro de estudio y como centro de trabajo. En este sentido, la Universidad tiene la responsabilidad de generar mecanismos de protección, denuncia y restitución de derechos para quienes enfrentan estas formas de discriminación.

- Las prácticas sexistas no son homogéneas. Las participantes dan cuenta de un amplio abanico de manifestaciones sexistas, desde algunas evidentes y grotescas, hasta otras más sutiles y toleradas. Esto da cuenta de la capacidad de adaptación $y$ versatilidad que tiene el sexismo para reproducir siempre su propósito más estructural, minorizar a las mujeres y lo femenino en el ámbito universitario.

Desde la perspectiva de las participantes es necesario que, tanto mujeres como hombres, aprendan a identificar las diferentes prácticas sexistas que se reproducen en el entorno académico para visibilizarlo y problematizarlo entre sus pares, pero también frente a quienes ejercen roles de poder en la institución.

Las mujeres - tanto desde su individualidad como desde espacios colectivos- 
han demostrado su capacidad de agencia y transformación, sin embargo, luchar en favor de la igualdad de oportunidades $y$ de condiciones justas para estudiar $y$ trabajar no es su responsabilidad exclusiva. En este sentido, la Universidad debe asumir un rol protagónico y comprometido en el combate de todas las formas de discriminación y sexismo que aún prevalecen en este centro de estudios.

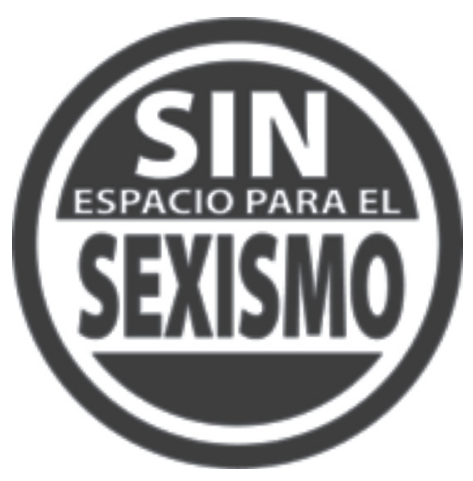

\section{REFERENCIAS}

Araya, S. (2003). Relaciones sexistas en la educación. Revista Educación, 27 (1). https:// revistas.ucr.ac.cr/index.php/educacion/ article/download/3805/3678/

Buss Thofehrn, M., López Montesinos, M., Rutz Porto, A., Coelho Amestoy, S., Oliveira Arrieira, I. y Mikla, M. (2013). Grupo focal: una técnica de recogida de datos en investigaciones cualitativas. Index de Enfermería, 22 (1-2), 75-78. https://dx.doi.org/10.4321/S113212962013000100016

Guizardi, M.L., Gonzálvez Torralbo, H. y Stefoni, C. (2019, en evaluación). The Shoemaker and her Barefooted Daughter. Narratives about Androcentrism and Gender Violence in Social Research. Frontiers: A Journal of Women Studies.

López, E. (2020). Violencia de género en adolescentes. Know and Share Psychology, I (1). Editorial Universidad de Almería. http://repositorio.ual.es/bitstream/
handle/10835/7810/2941-9234-1-PB. pdf? sequence $=1$

Maubrigades, S. (2020). Participación y segregación ocupacional de género en los sectores económicos de América Latina durante el siglo xx. América Latina en la Historia Económica, 27(3). DOI: 10.18232/alhe.1046

Mesa, S. (2019). Carreras académicas de mujeres en la Universidad de Costa Rica: Un reto para la igualdad. Revista Electrónica Actualidades Investigativas en Educación, 19 (1), 1-32. DOI: 10.15517/ aie.v19i1.35716

Mingo, A. y Moreno, H. (2017). Sexismo en la Universidad. Estudios Sociológicos, XXXV (105), 571-595. DOI: 10.24201/ es.2017v35n105.1434

Rodríguez, C. (2007). Economía del cuidado, equidad de género y nuevo orden económico internacional. Del Sur hacia el Norte: Economía política del orden económico internacional emergente (pp. 229-240). Clacso. http:// biblioteca.clacso.edu.ar/clacso/sursur/20100705083822/22RodriguezE.pdf

Sagot, M. (2018). La Universidad del Siglo XXI: Entre la igualdad, el sexismo y el mercado global del saber. [Ponencia] IX Coloquio Internacional: El potencial de las Instituciones de Educación Superior en la Construcción de una Sociedad Incluyente y Promotora de la Igualdad Social y de Género. Guanajuato, México.

Universidad de Costa Rica (UCR) (2017). Informe de brechas de género en recursos humanos en la UCR. Universidad de Costa Rica, San José, Costa Rica.

Universidad de Costa Rica (UCR) (31 de agosto de 2020). Reglamento de la Universidad de Costa Rica en contra del hostigamiento sexual.

Fecha de ingreso: 12/12/2019 Fecha de aprobación: 11/11/2020 
